\title{
Solutions to the Mixed Problem of Viscous Incompressible Flows in a Channel
}

\author{
Michal Beneš
}

\begin{abstract}
We study regularity of viscous incompressible fluid flows in a 2D channel with "do nothing" outflow boundary condition on the output for the steady Stokes and Navier-Stokes equations.
\end{abstract}

Mathematics Subject Classification (2000). 35Q30; 74G55; 74G20.

Keywords. Stokes and Navier-Stokes equations, Qualitative behavior of solutions, Local existence of solutions (near a given solution).

\section{Introduction}

Let $\Omega \in \mathcal{C}^{0,1}$ be a two-dimensional bounded domain. $\Omega$ represents a channel system filled up by a moving fluid. Let $\partial \Omega=\Gamma_{D} \cup \Gamma_{N}$ be such that $\Gamma_{D}$ and $\Gamma_{N}$ are closed, not necessarily connected $\left(\Gamma_{N}=\bigcup_{i}^{m} \Gamma_{N}^{(i)}, \Gamma_{N}^{(i)} \cap \Gamma_{N}^{(j)}=\emptyset\right.$ for $\left.i \neq j\right)$, the 1-dimensional measure of $\Gamma_{D} \cap \Gamma_{N}$ is zero and the 1-dimensional measure of $\Gamma_{D}$ is positive. Consider the mixed problem for the Navier-Stokes equations

$$
\begin{aligned}
-\nu \Delta \boldsymbol{u}+(\boldsymbol{u} \cdot \nabla) \boldsymbol{u}+\nabla \mathcal{P} & =\boldsymbol{f} & & \text { in } \Omega, \\
\nabla \cdot \boldsymbol{u} & =0 & & \text { in } \Omega, \\
\boldsymbol{u} & =\mathbf{0} & & \text { on } \Gamma_{D}, \\
-\mathcal{P} \boldsymbol{n}_{i}+\nu \frac{\partial \boldsymbol{u}}{\partial \boldsymbol{n}_{i}} & =F_{i} \boldsymbol{n}_{i} & & \text { on } \Gamma_{N}^{(i)}, i=1, \ldots, m,
\end{aligned}
$$

where $\boldsymbol{u}=\left(u_{1}, u_{2}\right)$ is velocity, $\nu$ denotes the kinematic viscosity ( $\nu$ is a positive constant), $\mathcal{P}$ represents pressure, $\boldsymbol{f}$ is a body force, $F_{i}$ are given constants and $\boldsymbol{n}_{i}=\boldsymbol{n}\left(\Gamma_{N}^{(i)}\right)$ is the outer unit normal to $\Gamma_{N}^{(i)}, i=1, \ldots, m$. We start with the

This outcome has been achieved with the financial support of the Ministry of Education, Youth and Sports of the Czech Republic, project No. 1M0579, within activities of the CIDEAS research centre. 
linearized problem, in which (1.2)-(1.4) hold and (1.1) is replaced with

$$
-\nu \Delta \boldsymbol{u}+\nabla \mathcal{P}=\boldsymbol{f} \quad \text { in } \quad \Omega .
$$

The problem (1.2)-(1.5) will be called steady Stokes problem with mixed boundary conditions.

There are many contributions dealing with the Dirichlet boundary conditions prescribed on $\Gamma_{D}$, which are used on the fixed walls of the channel. Let $\Gamma_{N}=$ $\bigcup \Gamma_{N}^{(i)}, \Gamma_{N}^{(i)}, i=1, \ldots, m$, denotes the open parts of the channel system. It is matter of discussion which boundary condition should be prescribed on the outlets of the channel system. We study here the boundary regularity of the solution to the mixed problem for the Navier-Stokes equations with the boundary condition (1.4) on the open parts of the channel system. The boundary condition (1.4), often called "do nothing" boundary condition, results from a variational principle and has been proven to be convenient in numerical modeling of parallel flows. Fore more informations about application of this boundary condition and the physical meaning of the quantities $F_{i}$ we refer to [1], [3], [4], [10]. The $\Gamma_{N}^{(i)}, i=1, \ldots, m$, are taken to be straight and orthogonal to the axis of the $i$-th outlets. Moreover, we assume that $\Gamma_{D}$ is smooth. These assumptions guarantee that singularities of the solutions can occur only in a neighborhood of boundary points, where the boundary conditions change. If corner points appear in $\Gamma_{D}$ we refer the interested reader to classical results for the Dirichlet problem by Kondrat'ev [5].

The existence of a unique solution of (1.1)-(1.4) was proved in [8] for data "near" values corresponding to already known solutions. In [7], the authors have formulated (1.1)-(1.4) as a variational inequality including the energy bound as a constraint. In the present paper, we calculate the regularity of solutions near points, where the boundary conditions change, under the assumption that the data of the problem are "small enough". Problem to prove the existence of global smooth solutions for general data (even in 2D) remains still open.

Remark 1.1. Usually, for steady flows, the quantities $F_{i}$ are prescribed constants. Since there is no influence of $F_{i}$ in (1.4) on the regularity of solutions, we will assume throughout this paper, without loss of generality, that $F_{i} \equiv 0, i=1, \ldots, m$.

\section{The linearized problem}

Denote by $V^{k, p}, k \in \mathbb{R}, 1<p<\infty$, the closure of

$$
\mathcal{E}(\Omega)=\left\{\boldsymbol{u} \in \mathcal{C}^{\infty}(\Omega)^{2} ; \quad \operatorname{div} \boldsymbol{u}=0, \quad \operatorname{supp} \boldsymbol{u} \subset \Omega, \quad \overline{\operatorname{supp} \boldsymbol{u}} \cap \Gamma_{D}=\emptyset\right\}
$$

in the norm $W^{k, p}(\Omega)^{2}$, where $\operatorname{supp} \boldsymbol{u}=\{\boldsymbol{x} \mid \boldsymbol{u}(\boldsymbol{x}) \neq \mathbf{0}\}$. When $p=2$ we use the standard notation $H^{k} \equiv W^{k, 2}(\Omega)$ and omit the domain $\Omega$ in the notation. Note that $V^{0,2}$ and $V^{1,2}$ are Hilbert spaces with scalar products

$$
(\boldsymbol{u}, \boldsymbol{v})=\int_{\Omega} \boldsymbol{u} \cdot \boldsymbol{v} \mathrm{d} \Omega \quad \text { and } \quad((\boldsymbol{u}, \boldsymbol{v}))=\int_{\Omega} \frac{\partial u_{i}}{\partial x_{j}} \frac{\partial v_{i}}{\partial x_{j}} \mathrm{~d} \Omega .
$$


If $\mathcal{X}$ is a Banach space, then $(\mathcal{X})^{*}$ represents its dual. Let $\boldsymbol{f} \in\left(V^{1,2}\right)^{*}$. A pair $(\boldsymbol{u}, \mathcal{P}) \in V^{1,2} \times L^{2}(\Omega)$ is called the weak solution of the problem (1.2)-(1.5) if $\nu((\boldsymbol{u}, \boldsymbol{v}))=\langle\boldsymbol{f}, \boldsymbol{v}\rangle$ for every $\boldsymbol{v} \in V^{1,2}$. The associated pressure $\mathcal{P}$ is a scalar function such that $\boldsymbol{u}$ and $\mathcal{P}$ satisfy the equation (1.5) in $\Omega$ in the sense of distributions. It is well known that there exists a uniquely determined weak solution $(\boldsymbol{u}, \mathcal{P})$ of the linearized problem (1.2)-(1.5) for every $\boldsymbol{f} \in\left(V^{1,2}\right)^{*}$.

We study regularity of the solution $(\boldsymbol{u}, \mathcal{P})$ near the corner point $A$ with corresponding angle $\omega_{A}=\frac{\pi}{2}$, where the boundary conditions change. The regularity problem in the cornered plane domain is solved using the weighted Sobolev spaces. Define $\mathcal{V}_{\beta}^{k, p}(\Omega), \beta \in \mathbb{R}$, as the closure of

$$
\mathcal{C}_{A}^{\infty}=\left\{\varphi \in \mathcal{C}^{\infty}(\Omega), \operatorname{supp} \varphi \text { bounded, } A \notin \operatorname{supp} \varphi\right\}
$$

with respect to the norm

$$
\|\varphi\|_{\mathcal{V}_{\beta}^{k, p}(\Omega)}=\left(\sum_{|\alpha| \leq k} \int_{\Omega} r^{p(\beta-k+|\alpha|)}\left|D^{\alpha} \varphi(\boldsymbol{x})\right|^{p} \mathrm{~d} \Omega\right)^{\frac{1}{p}}, \quad r=\operatorname{dist}(\boldsymbol{x}, A) .
$$

Note that the Stokes system with mixed boundary conditions (1.2)-(1.5) yields an elliptic boundary value problem in the sense of Agmon, Douglis and Nirenberg $(\mathrm{ADN})$. To achieve the regularity results we apply the general theory of ADN systems and follow the ideas of Kondrat'ev, who developed the "Fourier technique" for linear elliptic boundary value problems in domains with corner points.

Since the investigation of the regularity is a local problem, we will study the regularity of the weak solution near the corner points. After localizing the problem at each corner of the domain, i.e. identifying the origin of coordinates with the corner point, e.g. $A$, and multiplying the weak solution by a cut-off function $\eta(|\boldsymbol{x}|) \in \mathcal{C}^{\infty}\left(\mathbb{R}^{2}\right), 0 \leq \eta(|\boldsymbol{x}|)$,

$$
\eta(|\boldsymbol{x}|)=\left\{\begin{array}{lll}
1 & \text { for } & |\boldsymbol{x}|<\epsilon / 2 \\
0 & \text { for } & |\boldsymbol{x}|>\epsilon
\end{array}\right.
$$

one transforms the boundary value problem in a bounded domain to a boundary value problem in an infinite angle $\mathcal{K}$. By means of the change of coordinates $\left(x_{1}, x_{2}\right) \rightarrow(\omega, \xi)$, where $\xi=\log r,(\omega, r)$ are the polar coordinates with the origin $A$, we get the so called model problem in an infinite strip $\tilde{S}$. We apply the Fourier transform with respect to real variable $\xi \in \mathbb{R}$ to obtain the corresponding system of ordinary differential equations in variable $\omega$ with a complex parameter $\lambda, \widehat{\mathfrak{A}}\left(\omega, \partial_{\omega}, \lambda\right) \widehat{\mathfrak{u}}(\omega, \lambda)=\widehat{\mathfrak{f}}(\omega, \lambda)$ in the interval $I=\left(0, \frac{\pi}{2}\right)$. In the sequel we denote shortly $\widehat{\mathfrak{A}}(\lambda) \equiv \widehat{\mathfrak{A}}\left(\omega, \partial_{\omega}, \lambda\right)$. $\widehat{\mathfrak{A}}(\lambda)$ denotes the operator pencil which depends polynomially on the complex parameter $\lambda$. Solvability of the parameter dependent boundary value problems were studied in [6]. Roughly speaking, the operator $\widehat{\mathfrak{A}}(\lambda)$ is an isomorphism for all complex parameters $\lambda \in \mathbb{C}$ except at certain isolated points - the eigenvalues of $\widehat{\mathfrak{A}}(\lambda)$ (for precise definition of eigenvalues and corresponding eigensolutions we refer to [6], [9]). Consequently, the resolvent 
$\widehat{\mathfrak{R}}(\lambda)=\widehat{\mathfrak{A}}(\lambda)^{-1}$ is an operator-valued, meromorphic function of complex number $\lambda$ with poles of finite multiplicity. From the general theory of ADN systems and particular structure of the Stokes problem, we can derive the following proposition (see [5], [6] and [9]):

Theorem 2.1 (Regularity and expansion theorem). Let $A \in \partial \Omega$ be such a point on the boundary, where the boundary conditions change, $\beta_{1}$ and $\beta_{2}$ be real numbers satisfying the inequalities $\beta_{1}-1<\beta_{2}<\beta_{1},(\boldsymbol{u}, \mathcal{P}) \in \mathcal{V}_{\beta_{1}}^{l_{1}, 2}(\Omega)^{2} \times \mathcal{V}_{\beta_{1}}^{l_{1}-1,2}(\Omega)$ be the solution of (1.2)-(1.5) and $\boldsymbol{f} \in \mathcal{V}_{\beta_{2}}^{l_{2}-2, p}(\Omega)^{2} \cap \mathcal{V}_{\beta_{1}}^{l_{1}-2,2}(\Omega)^{2}, l_{2} \geq l_{1} \geq 2, \beta_{1} \geq \beta_{2} \geq 0$, $1<p<\infty$. Then the following propositions hold:

- If the strip $\operatorname{Im} \lambda \in\left[\beta_{2}+\frac{2}{p}-l_{2}, \beta_{1}+1-l_{1}\right]$ is free of eigenvalues of the operator pencil $\widehat{\mathfrak{A}}(\lambda)$, then $(\boldsymbol{u}, \mathcal{P}) \in \mathcal{V}_{\beta_{2}}^{l_{2}, p}(\Omega)^{2} \times \mathcal{V}_{\beta_{2}}^{l_{2}-1, p}(\Omega)$ and

$$
\|\boldsymbol{u}\|_{\mathcal{V}_{\beta_{2}}^{l_{2}, p}(\Omega)^{2}}+\|\mathcal{P}\|_{\mathcal{V}_{\beta_{2}}^{l_{2}-1, p}(\Omega)} \leq c\|\boldsymbol{f}\|_{\mathcal{V}_{\beta_{2}}^{l_{2}-2, p}(\Omega)^{2}}, \quad \text { where } \quad c=c(\nu, \Omega) .
$$

- Let $\lambda_{1}, \ldots, \lambda_{N_{0}}$ be the eigenvalues of $\widehat{\mathfrak{A}}(\lambda)$ in the strip $\operatorname{Im} \lambda \in\left[\beta_{2}+\frac{2}{p}-l_{2}, \beta_{1}+1-l_{1}\right]$. Then $(\boldsymbol{u}, \mathcal{P})$ admits in polar coordinates the asymptotic expansion

$$
(\boldsymbol{u}, \mathcal{P})=\eta(r)\left[\sum_{\mu=1}^{N_{0}} \sum_{\sigma=1}^{I_{\mu}} \sum_{k=0}^{k_{\mu_{\sigma}}-1} C_{\mu, \sigma, k} \boldsymbol{\Psi}_{\mu, \sigma, k}(r, \omega)\right]+\left[\overline{\boldsymbol{u}}_{r e g}(r, \omega), \overline{\mathcal{P}}_{r e g}(r, \omega)\right]
$$

in a neighborhood $W$ of the corner point $A$. The regular part of the solution $\left[\overline{\boldsymbol{u}}_{\text {reg }}(r, \omega), \overline{\mathcal{P}}_{\text {reg }}(r, \omega)\right] \in \mathcal{V}_{\beta_{2}}^{l_{2}, p}(W)^{2} \times \mathcal{V}_{\beta_{2}}^{l_{2}-1, p}(W), C_{\mu, \sigma, k}$ are constants depending on the data and the system of corresponding singular functions $\boldsymbol{\Psi}_{\mu, \sigma, k}$ is given by

$$
\boldsymbol{\Psi}_{\mu, \sigma, k}(r, \omega)=r^{i \lambda_{\mu}} \boldsymbol{\Upsilon}_{\mu, \sigma, k}(\log r, \omega)=r^{i \lambda_{\mu}} \sum_{q=0}^{k} \frac{(i \log r)^{q}}{q !} \boldsymbol{\Phi}_{\mu}^{\sigma, k-q}(\omega),
$$

where $\boldsymbol{\Phi}_{\mu}^{\sigma, k}$ is a canonical system of Jordan chains of $\widehat{\mathfrak{A}}(\lambda)$ with respect to eigenvalue $\lambda_{\mu} . I_{\mu, \sigma, k}=\{\mu, \sigma, k\}_{\mu=1, \ldots, N_{0}}^{\sigma=1, \ldots, I_{\mu}}, k=0, \ldots, k_{\mu_{\sigma}}-1, I_{\mu}=\operatorname{dim} \operatorname{ker} \widehat{\mathfrak{A}}\left(\lambda_{\mu}\right)$ denotes the geometrical multiplicity of $\lambda_{\mu}$, the number $k_{\mu_{\sigma}}$ denotes the length of the Jordan chain.

\subsection{Boundary value problem with parameter corresponding to the Stokes flows}

Stokes equations. Choose the origin $O$ at one of the corner points, e.g. $A$, and multiply the weak solution $(\boldsymbol{u}, \mathcal{P})$ by the cut off function $\eta$. Let $\boldsymbol{w}=\eta \boldsymbol{u}$ and $Q=\eta \mathcal{P}$. Further, denote by $\mathcal{K}$ an infinite angle with the vertex $O \equiv A$. Then

$$
\begin{aligned}
-\nu \Delta \boldsymbol{w}+\nabla Q & =\boldsymbol{g} \text { in } \mathcal{K}, \\
\nabla \cdot \boldsymbol{w} & =h \text { in } \mathcal{K},
\end{aligned}
$$

where

$$
\boldsymbol{g}=-\boldsymbol{u} \Delta \eta-2 \frac{\partial \boldsymbol{u}}{\partial x_{1}} \frac{\partial \eta}{\partial x_{1}}-2 \frac{\partial \boldsymbol{u}}{\partial x_{2}} \frac{\partial \eta}{\partial x_{2}}+\boldsymbol{f} \eta+(\nabla \eta) \mathcal{P}, \quad h=\boldsymbol{u} \cdot(\nabla \eta)
$$


with $\boldsymbol{g} \in L^{2}(\mathcal{K})^{2}$ and $h \in W^{1,2}(\mathcal{K}), \boldsymbol{w} \in W^{1,2}(\mathcal{K})^{2}, Q \in L^{2}(\mathcal{K})$. Rewrite the Stokes system $(2.3)-(2.4)$ using the polar coordinates $(r, \omega)$ and the substitution $r=e^{\xi}$ to the system of the form

$$
\begin{aligned}
-\nu\left(\frac{\partial^{2} \tilde{w}_{1}}{\partial \xi^{2}}+\frac{\partial^{2} \tilde{w}_{1}}{\partial \omega^{2}}\right)+\left(\frac{\partial \tilde{Q}}{\partial \xi}-\tilde{Q}\right) \cos \omega-\frac{\partial \tilde{Q}}{\partial \omega} \sin \omega & =\widetilde{G}_{1}(\xi, \omega), \\
-\nu\left(\frac{\partial^{2} \tilde{w}_{2}}{\partial \xi^{2}}+\frac{\partial^{2} \tilde{w}_{2}}{\partial \omega^{2}}\right)+\left(\frac{\partial \tilde{Q}}{\partial \xi}-\tilde{Q}\right) \sin \omega+\frac{\partial \tilde{Q}}{\partial \omega} \cos \omega & =\widetilde{G}_{2}(\xi, \omega), \\
\frac{\partial \tilde{w}_{1}}{\partial \xi} \cos \omega-\frac{\partial \tilde{w}_{1}}{\partial \omega} \sin \omega+\frac{\partial \tilde{w}_{2}}{\partial \xi} \sin \omega+\frac{\partial \tilde{w}_{2}}{\partial \omega} \cos \omega & =\widetilde{H}(\xi, \omega),
\end{aligned}
$$

that holds in an infinite strip $\tilde{S}=\left\{(\xi, \omega): \xi \in \mathbb{R}, 0<\omega<\frac{\pi}{2}\right\}, \tilde{\boldsymbol{w}}(\xi, \omega)=\boldsymbol{w}\left(x_{1}, x_{2}\right)$, $\tilde{Q}(\xi, \omega)=\sqrt{x_{1}^{2}+x_{2}^{2}} Q\left(x_{1}, x_{2}\right), \tilde{\boldsymbol{g}}(\xi, \omega)=\boldsymbol{g}\left(x_{1}, x_{2}\right), \tilde{h}(\xi, \omega)=h\left(x_{1}, x_{2}\right)$, $\widetilde{\boldsymbol{G}}(\xi, \omega)=e^{2 \xi} \tilde{\boldsymbol{g}}(\xi, \omega), \widetilde{H}(\xi, \omega)=e^{\xi} \tilde{h}(\xi, \omega)$. Remark that $\widetilde{\boldsymbol{G}} \in L^{2}(\tilde{S})^{2}$ and $\widetilde{H} \in W^{1,2}(\tilde{S})$.

Applying the complex Fourier transform $\mathcal{F}_{\xi \rightarrow \lambda}$ with respect to $\xi$, we get the following system of three ordinary differential equations depending on a complex parameter $\lambda$ with unknown functions $\widehat{w}_{1}, \widehat{w}_{2}$ and $\widehat{Q}$

$$
\begin{aligned}
-\nu\left(\frac{\partial^{2} \widehat{w}_{1}}{\partial \omega^{2}}+(\mathrm{i} \lambda)^{2}\left(\widehat{w}_{1}\right)\right)+(\mathrm{i} \lambda-1) \widehat{Q} \cos \omega-\frac{\partial \widehat{Q}}{\partial \omega} \sin \omega & =\widehat{G}_{1}(\lambda, \omega), \\
-\nu\left(\frac{\partial^{2} \widehat{w}_{2}}{\partial \omega^{2}}+(\mathrm{i} \lambda)^{2}\left(\widehat{w}_{2}\right)\right)+(\mathrm{i} \lambda-1) \widehat{Q} \sin \omega+\frac{\partial \widehat{Q}}{\partial \omega} \cos \omega & =\widehat{G}_{2}(\lambda, \omega), \\
(\mathrm{i} \lambda)\left(\widehat{w}_{1}\right) \cos \omega-\frac{\partial \widehat{w}_{1}}{\partial \omega} \sin \omega+(\mathrm{i} \lambda)\left(\widehat{w}_{2}\right) \sin \omega+\frac{\partial \widehat{w}_{2}}{\partial \omega} \cos \omega & =\widehat{H}(\lambda, \omega),
\end{aligned}
$$

that holds in the interval $I=\left(0, \frac{\pi}{2}\right), \widehat{\boldsymbol{G}}=\mathcal{F}_{\xi \rightarrow \lambda}(\widetilde{\boldsymbol{G}}), \widehat{H}=\mathcal{F}_{\xi \rightarrow \lambda}(\tilde{H})$, $\widehat{\boldsymbol{w}}=\mathcal{F}_{\xi \rightarrow \lambda}(\tilde{\boldsymbol{w}}), \widehat{Q}=\mathcal{F}_{\xi \rightarrow \lambda}(\tilde{Q})$. Remark that $\widehat{\boldsymbol{G}} \in L^{2}(I)^{2}$ and $\widehat{H} \in W^{1,2}(I)$. Denote by $\mathcal{A}(\lambda)$ the differential operator with complex parameter $\lambda$ corresponding to (2.5). Now (2.5) can be treated as the operator equation

$$
\mathcal{A}(\lambda)\left[\widehat{w}_{1}, \widehat{w}_{2}, \widehat{Q}\right]^{T}=\left[\widehat{G}_{1}, \widehat{G}_{2}, \widehat{H}\right]^{T} .
$$

Mixed boundary conditions. Introducing the polar coordinates and using the Fourier transform we modify the mixed boundary conditions to the transformed form

$$
\begin{aligned}
\nu \frac{\partial \widehat{w}_{1}}{\partial \omega}(\lambda, 0) & =0, & \nu \frac{\partial \widehat{w}_{2}}{\partial \omega}(\lambda, 0)-\widehat{Q}(\lambda, 0) & =0 \\
\widehat{w}_{1}\left(\lambda, \frac{\pi}{2}\right) & =0, & \widehat{w}_{2}\left(\lambda, \frac{\pi}{2}\right) & =0 .
\end{aligned}
$$

Denote by $\mathcal{B}(\lambda)$ the operator of the boundary conditions (2.6) and define the operator $\widehat{\mathfrak{A}}(\lambda)=[\mathcal{A}(\lambda), \mathcal{B}(\lambda)]$ corresponding to the mixed boundary value problem depending on the complex parameter $\lambda$ in the strong form

$$
\widehat{\mathfrak{A}}(\lambda): W^{2,2}(I)^{2} \times W^{1,2}(I) \rightarrow L^{2}(I)^{2} \times W^{1,2}(I) \times \mathbb{C}^{2} \times \mathbb{C}^{2} .
$$

The general solution. Denote by $\left[\hat{e}_{1}, \hat{e}_{2}, \hat{e}_{p}\right]^{T}$ the general solution of the system (2.5) with the vanishing right hand side, where $\hat{e}_{1}, \hat{e}_{2}$ stand for $\widehat{w}_{1}, \widehat{w}_{2}$ and $\hat{e}_{p}$ stands for $\widehat{Q}$, respectively. The general solution $\left[\hat{e}_{1}, \hat{e}_{2}, \hat{e}_{p}\right]^{T}$ of the equation

$$
\mathcal{A}(\lambda)\left(\hat{e}_{1}, \hat{e}_{2}, \hat{e}_{p}\right)^{T}=\mathbf{0}
$$


has the form $\left[\hat{e}_{1}, \hat{e}_{2}, \hat{e}_{p}\right]^{T}=\sum_{i=1}^{4} C_{i}(\lambda) \Phi_{i}(\lambda, \omega)$, where the fundamental system of the solution is given by

$$
\begin{array}{ll}
\Phi_{1}(\lambda, \omega)=\left(\begin{array}{c}
\cos (i \lambda \omega) \\
-\sin (i \lambda \omega) \\
0
\end{array}\right), & \Phi_{3}(\lambda, \omega)=\left(\begin{array}{c}
-\frac{i \lambda}{2} \cos [(i \lambda-2) \omega] \\
\sin (i \lambda \omega)+\frac{i \lambda}{2} \sin [(i \lambda-2) \omega] \\
-\nu 2 i \lambda \cos [(i \lambda-1) \omega]
\end{array}\right), \\
\Phi_{2}(\lambda, \omega)=\left(\begin{array}{c}
\sin (i \lambda \omega) \\
\cos (i \lambda \omega) \\
0
\end{array}\right), & \Phi_{4}(\lambda, \omega)=\left(\begin{array}{c}
\frac{i \lambda}{2} \sin [(i \lambda-2) \omega] \\
\cos (i \lambda \omega)+\frac{i \lambda}{2} \cos [(i \lambda-2) \omega] \\
\nu 2 i \lambda \sin [(i \lambda-1) \omega]
\end{array}\right),
\end{array}
$$

if $\lambda \neq 0$. Remark that $\lambda=0$ is not an eigenvalue of $\widehat{\mathfrak{A}}(\lambda)$.

Calculation of the eigenvalues of $\widehat{\mathfrak{A}}(\lambda)$. Substituting the general solution into the mixed boundary conditions (2.6) leads to the linear homogeneous system of four equations for four unknowns $C_{1}, C_{2}, C_{3}$ and $C_{4}$. The system admits a nontrivial solution if and only if the determinant of the corresponding matrix vanishes, i.e.

$$
D(\lambda)=\left|\begin{array}{cccc}
0 & 4-i \lambda & 0 & -2+i \lambda \\
2+i \lambda & 0 & 4+i \lambda & 0 \\
d_{31} & d_{32} & d_{33} & d_{34} \\
d_{41} & d_{42} & d_{43} & d_{44}
\end{array}\right|=0
$$

where

$$
\begin{array}{lll}
d_{31}=\cos \left(\frac{i \lambda \pi}{2}\right)-\frac{i \lambda}{2} \cos \left[(i \lambda-2) \frac{\pi}{2}\right], & d_{41}=\frac{i \lambda}{2} \sin \left[(i \lambda-2) \frac{\pi}{2}\right], \\
d_{32}=\sin \left(\frac{i \lambda \pi}{2}\right)-\frac{i \lambda}{2} \sin \left[(i \lambda-2) \frac{\pi}{2}\right], & d_{42}=-\frac{i \lambda}{2} \cos \left[(i \lambda-2) \frac{\pi}{2}\right], \\
d_{33}=-\frac{i \lambda}{2} \cos \left[(i \lambda-2) \frac{\pi}{2}\right], & d_{43}=\sin \left(\frac{i \lambda \pi}{2}\right)+\frac{i \lambda}{2} \sin \left[(i \lambda-2) \frac{\pi}{2}\right], \\
d_{34}=\frac{i \lambda}{2} \sin \left[(i \lambda-2) \frac{\pi}{2}\right], & d_{44}=\cos \left(\frac{i \lambda \pi}{2}\right)+\frac{i \lambda}{2} \cos \left[(i \lambda-2) \frac{\pi}{2}\right] .
\end{array}
$$

Calculation of the determinant (2.8) leads to the equation

$$
D(\lambda)=(i \lambda)^{2}-4 \cos ^{2}\left(\frac{i \lambda \pi}{2}\right)-\sin ^{2}\left(\frac{i \lambda \pi}{2}\right)=0 .
$$

The roots of the equation $D(\lambda)=0$ are the eigenvalues of $\widehat{\mathfrak{A}}(\lambda)$.

Remark 2.2. There are only two eigenvalues (of simple multiplicity) of the operator pencil $\widehat{\mathfrak{A}}(\lambda), \lambda_{0} \approx-1.352317 i$ and $\lambda_{1}=-i$, in the strip $\operatorname{Im} \lambda \in(-2, \delta)$, $\delta$ is a small positive real number. In the case $\operatorname{Im} \lambda_{1}=-1$ the solution is regular, the logarithmic term does not appear in the asymptotic expansion (2.2) (the singular part $r^{-\operatorname{Im} \lambda_{1}} \sum_{i=1}^{4} C_{i} \boldsymbol{\Phi}_{i}\left(\lambda_{0}, \omega\right)$ is smooth), hence if $\boldsymbol{f} \in L^{2}(\Omega)^{2}$, then $(\boldsymbol{u}, \mathcal{P}) \in\left(H^{2}\right)^{2} \times H^{1}$. Define the so called conjugate exponents $p_{0}$ and $\gamma_{0}$, $p_{0}=\frac{2}{\operatorname{Im} \lambda_{0}+2}(\approx 3.087930)$ and $\gamma_{0}=-1-\operatorname{Im} \lambda_{0}=\frac{p_{0}-2}{p_{0}}(\approx 0.352317)$.

\subsection{Regularity}

Let $(\boldsymbol{u}, \mathcal{P}) \in V^{1,2} \times L^{2}(\Omega)$ be the uniquely determined weak solution of (1.2)-(1.5) for given $\boldsymbol{f} \in\left(V^{1,2}\right)^{*}$. Note that the theory of Kondrat'ev developed in [5] yields $(\boldsymbol{u}, \mathcal{P}) \in \mathcal{V}_{1+\delta}^{2,2}(\Omega)^{2} \times \mathcal{V}_{1+\delta}^{1,2}(\Omega)$, where $\delta$ is a small positive real number. Let $p_{0}$ and $\gamma_{0}$ be conjugate exponents defined by Remark 2.2. Now, based on the Theorem 2.1 and Remark 2.2, we can formulate the regularity results for the Stokes problem $(1.2)-(1.5)$ : 
Corollary 2.3. Let $\boldsymbol{f} \in\left(H^{\gamma}\right)^{2}, 0 \leq \gamma<\gamma_{0}$, and let $(\boldsymbol{u}, \mathcal{P})$ be the weak solution of (1.2)-(1.5). Then $(\boldsymbol{u}, \mathcal{P})$ is contained in $\left(H^{2+\gamma}\right)^{2} \times H^{1+\gamma}$ and

$$
\|\boldsymbol{u}\|_{\left(H^{2+\gamma}\right)^{2}}+\|\mathcal{P}\|_{H^{1+\gamma}} \leq c\|\boldsymbol{f}\|_{\left(H^{\gamma}\right)^{2}}, \quad c=c(\nu, \Omega) .
$$

Corollary 2.4. Let $(\boldsymbol{u}, \mathcal{P})$ be the solution of $(1.2)-(1.5)$ and $\boldsymbol{f} \in L^{p}(\Omega)^{2}$.

- If $p<p_{0}$, then $(\boldsymbol{u}, \mathcal{P}) \in W^{1+\varepsilon, 2}(\Omega)^{2} \times W^{\varepsilon, 2}(\Omega) \cap W^{2, p}(\Omega)^{2} \times W^{1, p}(\Omega)$, where $\varepsilon=\min \{1,2-2 / p\}$, and the following estimate holds

$$
\|\boldsymbol{u}\|_{W^{2, p}(\Omega)^{2}}+\|\mathcal{P}\|_{W^{1, p}(\Omega)} \leq c\|\boldsymbol{f}\|_{L^{p}(\Omega)^{2}}, \quad c=c(\nu, \Omega) .
$$

- If $p \geq p_{0}$ then $(\boldsymbol{u}, \mathcal{P}) \in W^{\ell, p}(\Omega)^{2} \times W^{\ell-1, p}(\Omega) \cap W^{2, p_{0}-\delta}(\Omega)^{2} \times W^{1, p_{0}-\delta}(\Omega)$, with an arbitrary $\ell<2 / p-\operatorname{Im} \lambda_{0}$ and an arbitrary small positive real number $\delta$, admits the decomposition near the corner point $A$

$$
(\boldsymbol{u}, \mathcal{P})=[\overline{\boldsymbol{u}}(r, \omega), \overline{\mathcal{P}}(r, \omega)]=\eta(r)\left[r^{-\operatorname{Im} \lambda_{0}} \sum_{i=1}^{4} C_{i} \Phi_{i}\left(\lambda_{0}, \omega\right)\right]+\left[\overline{\boldsymbol{u}}_{r e g}(r, \omega), \overline{\mathcal{P}}_{r e g}(r, \omega)\right]
$$

where the regular part $\left[\overline{\boldsymbol{u}}_{\text {reg }}(r, \omega), \overline{\mathcal{P}}_{\text {reg }}(r, \omega)\right] \in W^{2, p}(\Omega)^{2} \times W^{1, p}(\Omega)$ has the maximal regularity allowed by the right hand side and the coefficients $C_{i}$ are determined by

$$
\begin{aligned}
& C_{1}=C_{0} \frac{-\operatorname{Im} \lambda_{0}+4}{2} \frac{\left(\operatorname{Im} \lambda_{0}+2\right) \sin \left(\operatorname{Im} \lambda_{0} \frac{\pi}{2}\right)-\operatorname{Im} \lambda_{0} \sin \left[\left(\operatorname{Im} \lambda_{0}+2\right) \frac{\pi}{2}\right]}{\left(-\operatorname{Im} \lambda_{0}+4\right) \cos \left(\operatorname{Im} \lambda_{0} \frac{\pi}{2}\right)+\operatorname{Im} \lambda_{0} \cos \left[\left(\operatorname{Im} \lambda_{0}+2\right) \frac{\pi}{2}\right]}, \\
& C_{2}=C_{0} \frac{\operatorname{Im} \lambda_{0}+2}{2}, \\
& C_{3}=C_{0} \frac{\left(\operatorname{Im} \lambda_{0}+2\right) \sin \left(\operatorname{Im} \lambda_{0} \frac{\pi}{2}\right)-\operatorname{Im} \lambda_{0} \sin \left[\left(\operatorname{Im} \lambda_{0}+2\right) \frac{\pi}{2}\right]}{\left(-\operatorname{Im} \lambda_{0}+4\right) \cos \left(\operatorname{Im} \lambda_{0} \frac{\pi}{2}\right)+\operatorname{Im} \lambda_{0} \cos \left[\left(\operatorname{Im} \lambda_{0}+2\right) \frac{\pi}{2}\right]}
\end{aligned}
$$

and $C_{4}=C_{0}$, where the coefficient $C_{0}=C_{0}(\nu, \Omega, \boldsymbol{f})$ (intensity factor) expresses the intensity of the singular part of the solution.

Proof. If $p<p_{0}$, then the strip $[2 / p-2, \delta)$ contains at most one eigenvalue $\lambda_{1}=-i$. Due to the Theorem 2.1 and Remark 2.2 the solution is regular. The estimate $(2.11)$ follows directly from Theorem 2.1. If $p \geq p_{0}$, then $(\boldsymbol{u}, \mathcal{P}) \in \mathcal{V}_{\beta_{0}}^{2, p}(\Omega)^{2} \times \mathcal{V}_{\beta_{0}}^{1, p}(\Omega)$, for all $\beta_{0}>2+\operatorname{Im} \lambda_{0}-2 / p$. Further,

$$
\mathcal{V}_{\beta_{0}}^{2, p}(\Omega)^{2} \times \mathcal{V}_{\beta_{0}}^{1, p}(\Omega) \hookrightarrow W^{2, \frac{2 p}{2+p \beta_{0}}}(\Omega)^{2} \times W^{1, \frac{2 p}{2+p \beta_{0}}}(\Omega) \hookrightarrow W^{2-\beta_{0}, p}(\Omega)^{2} \times W^{1-\beta_{0}, p}(\Omega),
$$

where we have used embeddings of weighted spaces [9] and standard Sobolev embeddings. Since $\beta_{0}>2+\operatorname{Im} \lambda_{0}-2 / p$, we can write $2 p /\left(2+p \beta_{0}\right)=p_{0}-\delta$, where $\delta$ is an arbitrary small positive real number and $\ell \equiv 2-\beta_{0}<2 / p-\operatorname{Im} \lambda_{0}$.

\section{The steady Navier-Stokes system}

We prove that the operator $\mathcal{N}$ corresponding to the Navier-Stokes problem is Fréchet differentiable at $\boldsymbol{u} \equiv \mathbf{0}$ and the Fréchet derivative $\mathcal{G}_{\mathbf{0}}=\left.D \mathcal{N}\right|_{\boldsymbol{u} \equiv \mathbf{0}}$ coincides with the Stokes problem. Applying the local diffeomorphism theorem, we prove that the solution of the Navier-Stokes problem has similar regularity as 
the solution of the Stokes problem if the norm of the given body force is "small enough".

Let $\boldsymbol{u}, \boldsymbol{v}, \boldsymbol{w} \in V^{1,2}$. Define a trilinear continuous form $b$ by

$$
b(\boldsymbol{u}, \boldsymbol{v}, \boldsymbol{w})=\int_{\Omega} u_{j} \frac{\partial v_{i}}{\partial x_{j}} w_{i} \mathrm{~d} \Omega .
$$

Definition 3.1. Let $\boldsymbol{f} \in\left(V^{1,2}\right)^{*}$. $\boldsymbol{u}$ is called the weak solution of (1.1)-(1.4), if $\boldsymbol{u}$ belongs to $V^{1,2}$ and

$$
\nu((\boldsymbol{u}, \boldsymbol{v}))+b(\boldsymbol{u}, \boldsymbol{u}, \boldsymbol{v})=(\boldsymbol{f}, \boldsymbol{v}) \quad \text { for all } \boldsymbol{v} \in V^{1,2} .
$$

Definition 3.2. Denote by $\mathscr{H}^{p}$ the closure of $\mathcal{E}(\Omega)$ in the norm $L^{p}(\Omega)^{2}$ and define the Banach space

$\mathscr{D}^{p}=\left\{\boldsymbol{\phi} ;\right.$ there exists $\boldsymbol{f} \in \mathscr{H}^{p}$ such that $\nu((\boldsymbol{\phi}, \boldsymbol{v}))=(\boldsymbol{f}, \boldsymbol{v})$ for every $\left.\boldsymbol{v} \in V^{1,2}\right\}$ with the norm $\|\boldsymbol{\phi}\|_{\mathscr{D}^{p}}=\|\boldsymbol{\phi}\|_{W^{2, \sigma}(\Omega)^{2}}, \sigma=\min \left\{p, p_{0}-\delta\right\}, \delta$ is an arbitrary small positive real number and $p_{0}$ denotes the conjugate exponent defined by Remark 2.2 .

Lemma 3.3. Let $\boldsymbol{\theta}, \boldsymbol{\psi} \in \mathscr{D}^{p}$. Then $b(\boldsymbol{\theta}, \boldsymbol{\psi},.) \subset \mathscr{H}^{p}$.

Proof. Note that Corollary 2.4 yields $\mathscr{D}^{p} \hookrightarrow \hookrightarrow L^{\infty}(\Omega)^{2}$ for all $p>1$. From the estimate

$$
|b(\boldsymbol{\theta}, \boldsymbol{\psi}, \boldsymbol{\phi})| \leq c\|\boldsymbol{\theta}\|_{L^{\infty}(\Omega)^{2}}\|\nabla \boldsymbol{\psi}\|_{V^{0, r}}\|\boldsymbol{\phi}\|_{V^{0, q}}, \quad c=c(\Omega),
$$

with an arbitrary $r \geq p, q=r /(r-1)$, we get, that $b(\boldsymbol{\theta}, \boldsymbol{\psi},$.$) maps \mathscr{D}^{p}$ into $\left(V^{0, q}\right)^{*}=V^{0, r} \hookrightarrow \mathscr{H}^{p}$.

Let $\boldsymbol{g} \in \mathscr{H}^{p}$. Then the Lax-Milgram theorem and Corollary 2.4 yield the existence of a uniquely determined $\boldsymbol{w} \in \mathscr{D}^{p}$, such that $\nu((\boldsymbol{w}, \boldsymbol{v}))=(\boldsymbol{g}, \boldsymbol{v})$ holds for all $\boldsymbol{v} \in V^{1,2}$. Define an operator $\mathcal{S}: \mathscr{D}^{p} \rightarrow \mathscr{H}^{p}$ by the equation

$$
(\mathcal{S}(\boldsymbol{w}), \boldsymbol{v})=\nu((\boldsymbol{w}, \boldsymbol{v})) \text { for all } \boldsymbol{v} \in V^{1,2} .
$$

Note that $\mathcal{S}: \mathscr{D}^{p} \rightarrow \mathscr{H}^{p}$ is the one-to-one mapping. Further, define an operator $\mathcal{N}: \mathscr{D}^{p} \rightarrow \mathscr{H}^{p}$ given by the equation

$$
(\mathcal{N}(\boldsymbol{u}), \boldsymbol{v})=(\mathcal{S}(\boldsymbol{u}), \boldsymbol{v})+b(\boldsymbol{u}, \boldsymbol{u}, \boldsymbol{v}) \quad \text { for all } \boldsymbol{v} \in V^{1,2} .
$$

Now (1.1)-(1.4) can be treated as one operator equation $\mathcal{N}(\boldsymbol{u})=\boldsymbol{f}$.

Let $\boldsymbol{u}$ be a fixed point in $\mathscr{D}^{p}$. Let $\mathcal{B} \boldsymbol{u}: \mathscr{D}^{p} \rightarrow \mathscr{H}^{p}$ be a linear operator given by the equation

$$
(\mathcal{B} \boldsymbol{u}(\boldsymbol{w}), \boldsymbol{v})=b(\boldsymbol{u}, \boldsymbol{w}, \boldsymbol{v})+b(\boldsymbol{w}, \boldsymbol{u}, \boldsymbol{v}) \quad \text { for all } \boldsymbol{v} \in V^{1,2} .
$$

Lemma 3.4. Let $\boldsymbol{u} \in \mathscr{D}^{p}$. The operator $\mathcal{G} \boldsymbol{u}$, given by the equation

$$
(\mathcal{G} \boldsymbol{u}(\boldsymbol{w}), \boldsymbol{v})=(\mathcal{S}(\boldsymbol{w}), \boldsymbol{v})+(\mathcal{B} \boldsymbol{u}(\boldsymbol{w}), \boldsymbol{v}) \quad \text { for all } \boldsymbol{v} \in V^{1,2},
$$

is the Fréchet derivative of $\mathcal{N}$ at the point $\boldsymbol{u}$ and $\mathcal{G} \boldsymbol{u} \in \mathcal{C}\left(\mathscr{D}^{p}\right)$. 
Proof. Since

$$
\|\mathcal{N}(\boldsymbol{u}+\boldsymbol{w})-\mathcal{N}(\boldsymbol{u})-\mathcal{G} \boldsymbol{u}(\boldsymbol{w})\|_{\mathscr{H}^{p}}=\|b(\boldsymbol{w}, \boldsymbol{w}, .)\|_{\mathscr{H}^{p}}
$$

and

$$
\|b(\boldsymbol{w}, \boldsymbol{w}, .)\|_{\mathscr{H}^{p}} \leq c\|\boldsymbol{w}\|_{\mathscr{D}^{p}}^{2} \quad \text { for all } \boldsymbol{w} \in \mathscr{D}^{p}
$$

we get

$$
\lim _{\|\boldsymbol{w}\|_{\mathscr{D} p} \rightarrow 0} \frac{\|\mathcal{N}(\boldsymbol{u}+\boldsymbol{w})-\mathcal{N}(\boldsymbol{u})-\mathcal{G} \boldsymbol{u}(\boldsymbol{w})\|_{\mathscr{H}^{p}}}{\|\boldsymbol{w}\|_{\mathscr{D}^{p}}} \leq \lim _{\|\boldsymbol{w}\|_{\mathscr{D} p} \rightarrow 0} c\|\boldsymbol{w}\|_{\mathscr{D}^{p}}=0 .
$$

The smoothness of $\mathcal{G} \boldsymbol{u} \in \mathcal{C}\left(\mathscr{D}^{p}\right)$ is obvious.

Lemma 3.5. Let $\boldsymbol{u} \equiv \mathbf{0}$. Then $\mathcal{G}_{\boldsymbol{u}} \equiv \mathcal{G}_{\mathbf{0}} \equiv \mathcal{S}$ is the one-to-one mapping.

Theorem 3.6 (Local Diffeomorphism Theorem, [2]). Let $\mathcal{X}$ and $\mathcal{Y}$ be Banach spaces, $L$ be a mapping from $\mathcal{X}$ into $\mathcal{Y}$ belonging to $\mathcal{C}^{1}$ in some neighborhood $V$ of point $\boldsymbol{u}_{0}$. If the Fréchet derivative $D L\left(\boldsymbol{u}_{0}\right): X \rightarrow Y$ is the one-to-one and onto $\mathcal{Y}$ and continuous mapping, then there exists a neighborhood $U$ of point $\boldsymbol{u}_{0}, U \subset V$ and a neighborhood $W$ of point $L\left(\boldsymbol{u}_{0}\right), W \subset \mathcal{Y}$, such that $L$ is the one-to-one mapping from $V$ onto $W$.

Theorem 3.7 (Main result). Let $\boldsymbol{f} \in \mathscr{H}^{p},\|\boldsymbol{f}\|_{\mathscr{H}^{p}}$ be "small enough". Then there exists a uniquely determined $\boldsymbol{u} \in \mathscr{D}^{p}$, such that

$$
\nu((\boldsymbol{u}, \boldsymbol{v}))+b(\boldsymbol{u}, \boldsymbol{u}, \boldsymbol{v})=(\boldsymbol{f}, \boldsymbol{v}) \quad \text { for all } \boldsymbol{v} \in V^{1,2}
$$

and there exists a constant $c$ such that

$$
\|\boldsymbol{u}\|_{\mathscr{D}^{p}}+\|\mathcal{P}\|_{W^{1, \sigma}(\Omega)} \leq c\|\boldsymbol{f}\|_{\mathscr{H}^{p}}, \quad c=c(\nu, \Omega) .
$$

$\sigma=\min \left\{p, p_{0}-\delta\right\}, \delta$ is an arbitrary small positive real number and $p_{0}$ denotes the conjugate exponent defined by Remark 2.2.

Proof. $\mathcal{G}_{\mathbf{0}}$ (a Fréchet derivative of $\mathcal{N}$ at the point $\boldsymbol{u} \equiv \mathbf{0}$ ) is the one-to-one mapping and onto $\mathscr{H}^{p}$. The continuity of $\mathcal{G}_{0}$ is obvious. Then the local diffeomorphism theorem yields the existence. Now we prove the estimate (3.4). For simplicity we denote by $c$ a generic constant, which can vary from line to line. For all $\boldsymbol{u} \in U \subset \mathscr{D}^{p}$ we have

$$
\boldsymbol{u}=D \mathcal{N}^{-1}(\mathbf{0})\left(\mathcal{N}(\boldsymbol{u})+\int_{0}^{1}(D \mathcal{N}(\mathbf{0})-D \mathcal{N}(s \cdot \boldsymbol{u})) \boldsymbol{u} \mathrm{d} s\right)
$$

and

$$
\begin{aligned}
\|\boldsymbol{u}\|_{\mathscr{D}^{p}} \leq & \left\|D \mathcal{N}^{-1}(\mathbf{0})\right\|_{\mathcal{L}\left(\mathscr{H}^{p}, \mathscr{D}^{p}\right)}\left(\|\mathcal{N}(\boldsymbol{u})\|_{\mathscr{H}^{p}}\right. \\
& \left.+\int_{0}^{1}\|D \mathcal{N}(\mathbf{0})-D \mathcal{N}(s \cdot \boldsymbol{u})\|_{\mathcal{L}\left(\mathscr{D}^{p}, \mathscr{H}^{p}\right)}\|\boldsymbol{u}\|_{\mathscr{D}^{p}} \mathrm{~d} s\right) \\
\leq & \left\|D \mathcal{N}(\mathbf{0})^{-1}\right\|_{\mathcal{L}\left(\mathscr{D}^{p}, \mathscr{H}^{p}\right)}\left(\|\boldsymbol{f}\|_{\mathscr{H}^{p}}+\frac{s^{\prime}}{2}\|\boldsymbol{u}\|_{\mathscr{D}^{p}}^{2}\right) \\
\leq & c\|\boldsymbol{f}\|_{\mathscr{H}^{p}}+\frac{1}{2}\|\boldsymbol{u}\|_{\mathscr{D}^{p}} .
\end{aligned}
$$


From (1.1) we have $\nabla \mathcal{P}=\boldsymbol{f}+\nu \Delta \boldsymbol{u}-(\boldsymbol{u} \cdot \nabla) \boldsymbol{u}$, hence,

$$
\begin{aligned}
\|\nabla \mathcal{P}\|_{L^{\sigma}(\Omega)^{2}} & \leq\|\boldsymbol{f}\|_{L^{\sigma}(\Omega)^{2}}+\nu\|\Delta \boldsymbol{u}\|_{L^{\sigma}(\Omega)^{2}}+\|(\boldsymbol{u} \cdot \nabla) \boldsymbol{u}\|_{L^{\sigma}(\Omega)^{2}} \\
& \leq c\left(\|\boldsymbol{f}\|_{L^{\sigma}(\Omega)^{2}}+\|\boldsymbol{u}\|_{V^{2, \sigma}}+\|\boldsymbol{u}\|_{V^{1, \sigma}}^{2}\right),
\end{aligned}
$$

$\sigma=\min \left\{p, p_{0}-\delta\right\}, \delta$ is an arbitrary small positive real number and $p_{0}$ denotes the conjugate exponent defined by Remark 2.2. Let $\|\boldsymbol{f}\|_{\mathscr{H}^{p}}$ be "small enough". Then (3.5) implies that (3.6) can be estimated by

$$
\|\nabla \mathcal{P}\|_{L^{\sigma}(\Omega)^{2}} \leq c\left(\|\boldsymbol{f}\|_{\mathscr{H}^{p}}+\|\boldsymbol{u}\|_{\mathscr{D}^{p}}\right) \text {. }
$$

(3.5) and (3.7) yield (3.4)

\section{References}

[1] P.M. Gresho: Incompressible fluid dynamics: some fundamental formulation issues, Ann. Rev. Fluid Mech. 23, 1991, 413-453.

[2] P.G. Ciarlet: Mathematical Elasticity, Vol. I. North-Holland, Amsterdam, 1988.

[3] G.P. Galdi, R. Rannacher, A.M. Robertson, S. Turek: Hemodynamical Flows. Modeling, Analysis and Simulation. Oberwolfach Seminars, Vol. 37. Birkhäuser, 2008.

[4] J.G. Heywood, R. Rannacher, and S. Turek. Artificial boundaries and flux and pressure conditions for the incompressible Navier-Stokes equations. Intern. J. Num. Meth. in Fluids, 22:325-352, 1996.

[5] V.A. Kondrat'ev: Boundary value problems for elliptic equations on domains with conical or angular points, Trudy Moskov. Mat. Obshch. 16(1967), Russian.

[6] V.A. Kozlov, V.G. Maz'ya, J. Rossmann: Elliptic Boundary Value Problems with Point Singularities, American Mathematical Society, 1997.

[7] S. Kračmar, J. Neustupa: A weak solvability of a steady variational inequality of the Navier-Stokes type with mixed boundary conditions. Nonlinear Analysis, Volume 47, Number 6, August 2001, pp. 4169-4180(12).

[8] P. Kučera: Solutions of the Stationary Navier-Stokes Equations with Mixed Boundary Conditions in Bounded Domain. Analysis, Numerics and Applications of Differential and Integral Equations, Vol. 379, Eds M. Bach, George C. Hsiao, A.M. Sändig, 1997, $127-131$.

[9] A. Kufner, A.M. Sändig: Some Aplications of Weighted Sobolev Spaces, TeubnerTexte zur Mathematik, Band 100, Leipzig 1987.

[10] R. Rannacher: Finite element methods for the incompressible Navier-Stokes equations, Fundamental Directions in Mathematical Fluid Mechanics (G. P. Galdi, et al., eds), 191-293, Birkhauser, Basel, 2000.

Michal Beneš

Czech Technical University in Prague,

Thákurova 7, 16629 Prague 6, Czech Republic

e-mail: benes@mat.fsv.cvut.cz 